\title{
An Integer Programming Model for Pricing American Contingent Claims under Transaction Costs
}

\author{
M. Ç. Pınar • A. Camcı
}

Accepted: 9 March 2010 / Published online: 23 March 2010

(C) Springer Science+Business Media, LLC. 2010

\begin{abstract}
We study the problem of computing the lower hedging price of an American contingent claim in a finite-state discrete-time market setting under proportional transaction costs. We derive a new mixed-integer linear programming formulation for calculating the lower hedging price. The linear programming relaxation of the formulation is exact in frictionless markets. Our results imply that it might be optimal for the holder of several identical American claims to exercise portions of the portfolio at different time points in the presence of proportional transaction costs while this incentive disappears in their absence.
\end{abstract}

Keywords American Contingent Claims - Transaction Costs · Mixed-integer Programming · Linear Programming · Martingales · Incomplete Markets · Pricing · Hedging $\cdot$ Dividends

\section{Introduction}

The purpose of the present note is to examine, using integer programming, the problem of computing a fair price (in the sense of not allowing arbitrage) for the holder (buyer) of an American contingent claim (ACC) in a discrete-time, finite state incomplete market model where the stock trades incur transaction costs proportional to the magnitude to the trade. Since American contingent claims allow the holder to exercise the claim at any point during its lifetime as opposed to their European counterparts which can only be exercised at maturity, the computation of a fair price also involves

M. Ç. Pınar $(\varangle)$. A. Camcı

Department of Industrial Engineering, Bilkent University, 06800 Ankara, Turkey e-mail: mustafap@bilkent.edu.tr

A. Camc1

e-mail: camci@bilkent.edu.tr 
the choice of an optimal exercise strategy, which opens the way to modeling with binary variables. King (2002) showed the connections between linear programming and modern techniques of contingent claim pricing in mathematical finance in the context of European claims. The main contribution of the present note is to further the bond between finite dimensional optimization and mathematical finance by adding an integer programming model to the list of finite-dimensional optimization approaches useful for pricing contingent claims in financial markets.

It is well-known that a fair price for the buyer (lower hedging price) of an American contingent claim, given for the first time in Harrison and Kreps (1979), is computed as the maximum over a set of stopping times of the minimum of discounted expected pay-off at the point of stopping over all martingale measures for the buyer of the American claim, and the maximization over a set of stopping times of the maximum of discounted expected pay-off at the point of stopping over all martingale measures for the seller of the American claim. No-arbitrage pricing of American claims was first studied by Bensoussan (1984) and Karatzas (1988) for complete markets in continuous time. A good reference for continuous time pricing of American contingent claims is Detemple (2005); see also the survey by Myeni (1992). The book by Föllmer and Schied (2004) contains a thorough discussion of pricing and hedging American claims in discrete time but infinite state space setting. A derivation of these formulae in a discrete-time, finite state probability context can be found in Chalasani and Jha (2001).

In the presence of transaction costs proportional to the magnitude of the stock trades it is usually the case that perfect replication is impossible, and therefore the markets become incomplete. Koehl et al. (1999), Jaschke (1996) and Ortu (2001) study ACCs in discrete time, while Karatzas and Kou (1998) study no-arbitrage pricing and hedging of ACCs in continuous time under portfolio constraints, and Buckdahn and $\mathrm{Hu}$ (1998) consider jump diffusions for the stock price process in a similar context. Davis and Zariphopoulou (1995) study utility maximization for pricing American claims. Bouchard and Temam (2005) extend and generalize the discrete-time results of Chalasani and Jha for the upper hedging price to general discrete time markets in an infinite state space setting. In a separate line of work, Tokarz and Zastawniak (2006) develop efficient dynamic programming algorithms for pricing American options in discrete time under small transaction costs, and Roux and Zastawniak (2006) extend previous work by removing the restriction on transactions costs. It is important to note that Roux and Zastawniak (2006) allow a revision of portfolio positions before new prices are revealed. This feature of their formulation enables them to work with path independent portfolio and exercise strategies. However, as illustrated and discussed in Edirisinghe et al. (1993), path independent strategies can be sub-optimal hedging strategies in the presence of transaction costs. Our models in the present paper allow a revision of the portfolio (and exercise) only after new prices are revealed, and are based on path dependent strategies.

In Chalasani and Jha (2001), Bouchard and Temam (2005) and Pennanen and King (2006), the seller price (the upper hedging price) is thoroughly studied. In the present paper, we focus on the lower hedging problem and give for the first time (to the best of the authors' knowledge) an integer programming formulation for computing the lower hedging price, departing from a max-min expression of Chalasani and Jha for 
the lower hedging price. Then we exhibit a numerical example showing that a linear relaxation might lead to a non-zero duality gap, contrary to a claim made in Chalasani and Jha (2001). This result implies that it might be optimal for the holder of several identical ACCs to exercise them partially at different time points. For frictionless markets, the linear programming relaxation is exact. Hence, there is no incentive for the holder of ACCs not facing transaction costs to exercise them partially. The formulation is easily extended to allow dividend paying stocks.

\section{Preliminaries}

Throughout the paper, we refer to the optimal value of an optimization problem $P$ as opt $(P)$. We assume as in Chalasani and Jha (2001), King (2002) that security prices and other payments are discrete random variables supported on a finite probability space $(\Omega, \mathcal{F}, \mathbb{P})$ whose atoms are sequences of real-valued vectors (asset values) over discrete time periods $t=0,1, \ldots, T$. The market evolves as a discrete, nonrecombinant scenario tree (hence, suitable for incomplete markets; see Edirisinghe et al. (1993) for a discussion) in which the partition of probability atoms $\omega \in \Omega$ generated by matching path histories up to time $t$ corresponds one-to-one with nodes $n \in \mathcal{N}_{t}$ at level $t$ in the tree. The set $\mathcal{N}_{0}$ consists of the root node $n=0$, and the leaf nodes $n \in \mathcal{N}_{T}$ correspond one-to-one with the probability atoms (also referred to as paths or scenarios) $\omega \in \Omega$. We use $\omega_{t}$ to mean the node at time $t$ on path $\omega$. In the scenario tree, every node $n \in \mathcal{N}_{t}$ for $t=1, \ldots, T$ has a unique parent denoted $\pi(n) \in \mathcal{N}_{t-1}$, and every node $n \in \mathcal{N}_{t}, t=0,1, \ldots, T-1$ has a non-empty set of child nodes $\mathcal{C}(n) \subset \mathcal{N}_{t+1}$. We denote the set of all nodes in the tree by $\mathcal{N}$, and the set of all nodes except the root node and the leaf nodes by $\overline{\mathcal{N}}$. The set $\mathcal{A}(n)$ denotes the collection of ascendant nodes or the unique path leading to node $n$ (excluding itself) from node 0 . We also use the notation $t(n)$ to denote the time period that the node $n$ belongs to, $\mathcal{D}(n)$ for all descendants of node $n$ (including node $n$ itself). The probability distribution $\mathbb{P}$ is obtained by attaching positive weights $p_{n}$ to each leaf node $n \in \mathcal{N}_{T}$ so that $\sum_{n \in \mathcal{N}_{T}} p_{n}=1$. For each non-leaf (intermediate level) node in the tree we have, recursively,

$$
p_{n}=\sum_{m \in \mathcal{C}(n)} p_{m}, \quad \forall n \in \mathcal{N}_{t}, \quad t=T-1, \ldots, 0 .
$$

Hence, each non-leaf node has a probability mass equal to the combined mass of its child nodes.

A random variable $X$ is a real valued function defined on $\Omega$. It can be lifted to the nodes of a partition $\mathcal{N}_{t}$ of $\Omega$ if each level set $\left\{X^{-1}(a): a \in \mathbb{R}\right\}$ is either the empty set or is a finite union of elements of the partition. In other words, $X$ can be lifted to $\mathcal{N}_{t}$ if it can be assigned a value on each node of $\mathcal{N}_{t}$ that is consistent with its definition on $\Omega$. This kind of random variable is said to be measurable with respect to the information contained in the nodes of $\mathcal{N}_{t}$, or equivalently, $\mathcal{F}_{t}$-measurable. A stochastic process $\left\{X_{t}\right\}$ is a time-indexed collection of random variables such that each $X_{t}$ is measurable with respect $\mathcal{N}_{t}$. While not needed in the finite probability setting, the 
$\sigma$-algebras $\mathcal{F}_{t}$ generated by the partitions $\mathcal{N}_{t}$ are such that, $\mathcal{F}_{0}=\{\emptyset, \Omega\}, \mathcal{F}_{t} \subset \mathcal{F}_{t+1}$ for all $0 \leq t \leq T-1$ and $\mathcal{F}_{T}=\mathcal{F}$. A decision process is said to be $\left(\mathcal{F}_{t}\right)_{t=0^{-}}^{T}$ adapted if for each $t=0, \ldots, T$, the decision depends on the element of $\mathcal{F}_{t}$ that has been realized at stage $t$. The expected value of $X_{t}$ is uniquely defined by the sum $\mathbb{E}^{\mathbb{P}}\left[X_{t}\right]:=\sum_{n \in \mathcal{N}_{t}} p_{n} X_{n}$. The conditional expectation of $X_{t+1}$ on $\mathcal{N}_{t}$ is given by the expression $\mathbb{E}^{\mathbb{P}}\left[X_{t+1} \mid \mathcal{N}_{t}\right]:=\sum_{m \in \mathcal{C}(n)} \frac{p_{m}}{p_{n}} X_{m}$.

The market consists of a riskless asset (cash account) and a risky security with prices at node $n$ given by the scalar $S_{n}$. We assume the cash account appreciates in value by a factor $R \geq 1$ in each period. Transaction costs are modeled as follows: at node $n$, selling one share of stock the investor gets $S_{n}(1-\mu)$, and has to disburse $S_{n}(1+\lambda)$ upon acquisition of one share of stock. Our choice of two instruments is by no means a limitation of our models, and all the development in the paper can be re-iterated for a financial market with several risky securities and a claim with pay-off contingent on the values of several securities.

An ACC $F$ is a financial instrument generating a real-valued stochastic (cash-flow) process $\left(F_{t}\right)_{t=0, \ldots, T}$. At any stage $t=0, \ldots, T$, the holder of an ACC may decide to take $F_{t}$ in cash and terminate the process. Using this definition, an American call option on a stock $S$ with strike price $K$ corresponds to $F=S-K$. American put is obtained by reversing the sign of $F$. We can define a European call option with maturity $T$ by setting $F_{t}=0$ for $t \neq T$. Bermudan call options having exercise date set $T^{\prime} \subset\{1, \ldots, T\}$ can be defined by setting $F_{t}=0$ for $t \notin T^{\prime}$. In our finite probability space setting an American contingent claim $F$ generates payoff opportunities $F_{n},(n \geq 0)$ to its holder depending on the states $n$ of the market.

A stopping time $\tau$ is a random variable that maps each scenario (path) $\omega \in \Omega$ to a number $[0,1 \ldots, T]$, with the restriction that for any $t$, the indicator random variable $\mathbb{I}_{\tau=t}$ is $\mathcal{F}_{t}$-measurable. In other words, if there is some path $\omega$ with $\tau(\omega)=t$, and $\omega_{t}=u$, then for every path $\omega^{\prime}$ containing $u$, we must have $\tau\left(\omega^{\prime}\right)=t$. Therefore, for any path $\omega$, there is a unique stopping point $\omega_{\tau(\omega)}$. For any adapted process $\left\{X_{t}\right\}$, we denote by $X_{\tau}$ the random variable that maps a path $\omega \in \Omega$ to $X_{\tau(\omega)}(\omega)$, where the notation $X_{t}(\omega)$ refers to the value of $X$ at time $t$ on path $\omega$. Let $\mathcal{T}$ be the set of all stopping times.

For any probability measure $\mathbb{P}$ and exercise strategy (stopping time) $\tau$, we say that $\mathbb{P}$ is a $(\lambda, \mu, \tau)$-approximate martingale measure, if $\mathbb{P}$-almost surely,

$$
S_{t}^{*}(1-\mu) \leq \mathbb{E}^{\mathbb{P}}\left[S_{\tau}^{*} \mid \mathcal{N}_{t}\right] \leq S_{t}^{*}(1+\lambda) \forall t<\tau
$$

where $S_{t}^{*}$ denotes the discounted stock price $S_{t} R^{-t}$. We use $\mathcal{P}(\lambda, \mu, \tau)$ to denote the set of all $(\lambda, \mu, \tau)$-approximate martingale measures.

The buyer's objective is to compute the largest amount it can borrow to acquire the claim while picking a suitable exercise time for the claim and covering this debt by self-financing portfolio transactions in the financial market (here represented by cash and the risky asset) using the proceeds from the claim at the chosen date of exercise. In other words, the buyer's strategy is to find the maximum amount, $x^{*}$ say, he/she can borrow (by short selling stock) to acquire the claim and with the remaining cash to initiate a self-financing, adapted portfolio trading strategy and a stopping time (exercise 
strategy) $\tau$ such that at time $\tau$ the value of the portfolio and the pay-off from the claim are sufficient to close all short positions to avoid any losses. The buyer has to enforce this strategy over all paths. It is clear (see also Theorem 8.2 Chalasani and Jha 2001) that if the buyer can acquire the claim for a price inferior to $x^{*}$, then this constitutes an arbitrage opportunity for the buyer as follows. The buyer still borrows $x^{*}$, acquires the claim for a price $p<x^{*}$, ending up with the difference $x^{*}-p$ at time 0 , follows the optimal self-financing portfolio strategy and the exercise strategy to repay the debt in all states of the world; see Chalasani and Jha (2001).

Since for a fixed exercise strategy, the valuation of the claim can be expressed as an expectation using convex duality theory, the following max-min expression for the lower hedging price $h_{\text {low }}(\lambda, \mu, F)$ of an ACC $F$ was given in Theorem 12.2 of Chalasani and Jha (2001):

$$
h_{\text {low }}(\lambda, \mu, F)=\max _{\tau \in \mathcal{T}} \min _{\mathbb{P} \in \mathcal{P}(\lambda, \mu, \tau)} \mathbb{E}^{\mathbb{P}}\left[F_{\tau}^{*}\right]
$$

where $F_{t}^{*}$ denotes the discounted ACC pay-off $F_{t} R^{-t}$. This price is finite if and only if the market is arbitrage free in the sense of Chalasani and Jha (see definition on Chalasani and Jha 2001, p. 53 and Theorem 13.1), which we assume to be the case in the sequel.

Three assumptions are made in Chalasani and Jha (2001): (a) debt must be repaid in cash, (b) no transaction cost is incurred when a portfolio is liquidated to settle a debt, and (c) no new portfolio positions are taken at period $T$. It is clearly the case that Chalasani and Jha are interested in path dependent portfolio and exercise strategies which we also adopt. The numerical example at the opening of Sect. 3 below illustrates the importance of this point.

\section{The Formulation}

Consider a two period example in Fig. 1 where we assume for simplicity that the cash account does not generate any interest. The numbers next to nodes in the tree are the stock prices. The stock price is initially 10 at $t=0$. It either goes up to 15 or down 7 at $t=1$ with some probabilities. If it is equal to 15 at $t=1$, then either it goes up to 18 or down to 14 at $t=2$. If it is equal to 7 at $t=1$, then either it goes up to 13 or down to 4 at $t=2$. This gives a non-recombinant stochastic tree with node 0 as the root, node 1 (up to 15) and node 2 (down to 7) at $t=1$. At $t=2$, from node 1 , the tree evolves to either node 3 (up to 18 from 15) or to node 4 (down to 14 from 15); from node 2 it evolves to either node 5 (up to 13 from 7 ) or to node 6 (down to 4 from 7 ). We assume $\lambda=\mu=0.01$. We want to calculate the lower hedging price of an American call option with strike price equal to 10 (an at-the-money American call).

Using (2) and evaluating different possibilities, the optimal value accurate to six digits is 2.435125 and attained using the following optimal exercise strategy: exercise if the stock price evolves to node 1 at $t=1$, exercise at $t=2$ if the stock price evolves to node 5. Notice that the optimal strategy is a path dependent exercise strategy. In fact, the two path independent exercise strategies that are of interest in this example, 
Fig. 1 A numerical example for $P^{1}(0.01,0.01)$

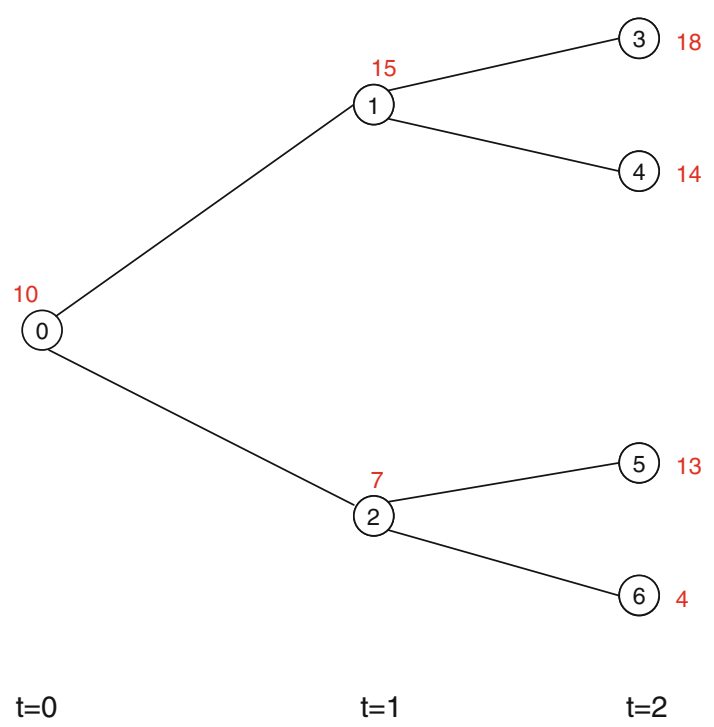

e.g., exercise only at $t=1$ or only at time $t=2$ are both sub-optimal with objective function values 1.812500 and 2.415296 , respectively.

Now, we derive a formulation for the lower hedging price. First let us deal with the inner minimization for a fixed exercise strategy that is treated as a constant. We use binary variables $e_{n}$ for exercise decisions, i.e., the ACC is exercised at node $n$ if $e_{n}=1$, and is not exercised at node $n$ if $e_{n}=0$. Since the ACC can only be exercised once over each path (scenario) in the tree, one has to enforce the restriction:

$$
\sum_{m \in \mathcal{A}(n) \cup\{n\}} e_{m} \leq 1, \quad \forall n \in \mathcal{N}_{T}
$$

The above is in one-to-one correspondence with the stopping time definitions in Sect. 2. We use $E$ to denote the set of all binary valued $e_{n}, n \in \mathcal{N}$ satisfying (3).

Now, for a given set of fixed values $e_{n}^{*}$ for $e_{n}, n \in \mathcal{N}$ respecting the above restriction (3), since the optimal exercise strategy is a not necessarily a path independent strategy, we must allow for the possibility that all time periods $1, \ldots, T$ are eligible to be picked as the stopping time $\tau$ over a given path as long as there is at most one exercise period over all paths. Therefore, we express the inner minimization problem in (2) taking into account all exercise possibilities as:

$$
\min _{q_{n}, n \in \mathcal{N}} \sum_{n \in \mathcal{N} \backslash\{0\}} q_{n} e_{n}^{*} F_{n}^{*}+e_{0}^{*} F_{0}
$$

subject to the restrictions

$q_{n} S_{n}^{*}(1-\mu) \leq \sum_{m \in \mathcal{D}\left(n, t^{\prime}\right)} q_{m} S_{m}^{*} \leq q_{n} S_{n}^{*}(1+\lambda) \forall n \in \mathcal{N}_{t}, \forall t<t^{\prime}$, and $t^{\prime} \in[1, \ldots, T]$ 


$$
\begin{aligned}
& q_{n}=\sum_{m \in \mathcal{C}(n)} q_{m} \forall n \in \mathcal{N}_{t}, \quad \forall t \in[0, \ldots, T-1], \\
& q_{0}=1, \quad \text { and } \quad q_{n} \geq 0, \quad \forall n \in \mathcal{N}_{T} .
\end{aligned}
$$

Let $\mathcal{Q}(\lambda, \mu)$ denote the set of probability measures $\mathbb{Q}=\left\{q_{n}\right\}_{n \in \mathcal{N}}$ satisfying the above constraints. Hence, we can rewrite expression (2) as:

$$
\max _{e \in E} \min _{\mathbb{Q} \in \mathcal{Q}(\lambda, \mu)} \sum_{n \in \mathcal{N} \backslash\{0\}} q_{n} e_{n} F_{n}^{*}+e_{0} F_{0} .
$$

Now, attaching Lagrange multipliers $b_{0}$ to the last constraint, $b_{n}$ to each of the second set of constraints for $n \in \mathcal{N} \backslash\{0\}$, and (non-negative) $d_{n}^{t^{\prime}}$ and $u_{n}^{t^{\prime}}$ to each of the first set of constraints, we obtain the Lagrange function

$$
\begin{aligned}
L\left(q_{n}, b_{n}, u_{n}^{t^{\prime}}, d_{n}^{t^{\prime}}\right)= & \sum_{n \in \mathcal{N} \backslash\{0\}} q_{n} e_{n}^{*} F_{n}^{*}+e_{0}^{*} F_{0} \\
& +\sum_{t^{\prime}=1}^{T} \sum_{t<t^{\prime}} \sum_{n \in \mathcal{N}_{t}} d_{n}^{t^{\prime}}\left[q_{n} S_{n}^{*}(1-\mu)-\sum_{m \in \mathcal{D}\left(n, t^{\prime}\right)} q_{m} S_{m}^{*}\right] \\
& +\sum_{t^{\prime}=1}^{T} \sum_{t<t^{\prime}} \sum_{n \in \mathcal{N}_{t}} u_{n}^{t^{\prime}}\left[\sum_{m \in \mathcal{D}\left(n, t^{\prime}\right)} q_{m} S_{m}^{*}-q_{n} S_{n}^{*}(1+\lambda)\right] \\
& +\sum_{n \in \mathcal{N} \backslash\{0\}} b_{n}\left(q_{n}-\sum_{m \in \mathcal{C}(n)} q_{m}\right)+b_{0}\left(\sum_{m \in \mathcal{C}(0)} q_{m}-1\right) .
\end{aligned}
$$

and are ready to compute the dual problem through

$$
\max _{b_{n}, u_{n}^{t^{\prime}}, d_{n}^{t^{\prime}}} \min _{q_{n}} L\left(q_{n}, b_{n}, u_{n}^{t^{\prime}}, d_{n}^{t^{\prime}}\right)
$$

After rearranging and minimizing the Lagrange function separately over each $q_{n} \geq 0$ for all $n \in \mathcal{N}$ we obtain the Lagrange dual problem

$$
\begin{gathered}
\max \quad-b_{0}+\sum_{t=1}^{T}\left(d_{0}^{t} S_{0}(1-\mu)-u_{0}^{t} S_{0}(1+\lambda)\right)+e_{0}^{*} F_{0} \\
\text { s.t. } \quad b_{n} \leq R b_{\pi(n)}+e_{n}^{*} F_{n}+\sum_{m \in \mathcal{A}(n)} S_{n}\left(u_{m}^{t(n)}-d_{m}^{t(n)}\right) \\
\quad+\sum_{t=t(n)+1}^{T} S_{n}\left((1-\mu) d_{n}^{t}-(1+\lambda) u_{n}^{t}\right), \forall n \in \overline{\mathcal{N}}, \\
0 \leq b_{\pi(n)}+e_{n}^{*} F_{n}+\sum_{m \in \mathcal{A}(n)} S_{n}\left(u_{m}^{T}-d_{m}^{T}\right), \forall n \in \mathcal{N}_{T}
\end{gathered}
$$


with the non-negativity constraints on all the variables $u_{n}^{t}, d_{n}^{t}$, for all $n \in \mathcal{N}$ and all $t \in[0,1, \ldots, T]$.

The above problem combined with the outer maximization over $e \in E$ yields the problem $P^{1}(\lambda, \mu)$

$$
\begin{aligned}
& \max \quad-b_{0}+ \sum_{t=1}^{T}\left(d_{0}^{t} S_{0}(1-\mu)-u_{0}^{t} S_{0}(1+\lambda)\right)+e_{0} F_{0} \\
& \text { s.t. } \quad b_{n} \leq R b_{\pi(n)}+e_{n} F_{n}+\sum_{m \in \mathcal{A}(n)} S_{n}\left(u_{m}^{t(n)}-d_{m}^{t(n)}\right) \\
&+\sum_{t=t(n)+1}^{T} S_{n}\left((1-\mu) d_{n}^{t}-(1+\lambda) u_{n}^{t}\right), \quad \forall n \in \overline{\mathcal{N}}, \\
& 0 \leq R b_{\pi(n)}+e_{n} F_{n}+\sum_{m \in \mathcal{A}(n)} S_{n}\left(u_{m}^{T}-d_{m}^{T}\right), \forall n \in \mathcal{N}_{T} \\
& 1 \geq \sum_{\substack{m \in \mathcal{A}(n) \cup\{n\} \\
e_{n} \in}} e_{m} \forall n \in \mathcal{N} T \\
&\{0,1\}, \forall n \in \mathcal{N}
\end{aligned}
$$

and the non-negativity constraints on all the variables $u_{n}^{t}, d_{n}^{t}$, for all $n \in \mathcal{N}$ and all $t \in[0,1, \ldots, T]$.

Hence, we have proved the following.

Theorem $1 h_{\text {low }}(\lambda, \mu, F)=\operatorname{opt}\left(P^{1}(\lambda, \mu)\right)$.

This problem has a very clear hedging interpretation. We view the non-negative variable $u_{n}^{t}$ as a long position in the risky asset acquired at node $n$ for liquidation at time period $t$. Similarly we let non-negative variable $d_{n}^{t}$ denote a short position in the risky asset open at node $n$ to be closed at time period $t$. We view $b_{n}$ as the cash position at node $n$. The first set of constraints express the following balance requirement for each "interior" (non-leaf nodes also excluding the root node) node: cash available from the parent node (magnified by the interest) plus pay-off from the option in case of exercise and proceeds from short sales after accounting for transaction costs, and proceeds from liquidation of earlier long positions (without incurring transaction costs) should be sufficiently large to balance new long positions destined for liquidation in future time points (with transaction costs) and closing of short positions earlier established at no transaction cost. A similar interpretation holds for the leaf nodes where no transaction costs are involved, since no new positions are acquired. These hedging constraints are in one-to-one correspondence with the hedging strategy of the buyer as announced on Chalasani and Jha (2001, pp. 52-53): the buyer starts out by borrowing a certain amount at time 0 to acquire the $\mathrm{ACC}$, and chooses a path dependent exercise strategy from which he/she obtains a certain pay-off with which to close his/her initial debt.

Now, let us return to the numerical example introduced at the beginning of this section. When we solve the problem as a mixed-integer programming problem we obtain the following hedging strategy: short sell 0.502917 shares of stock at time $t=0$ to be closed (without transaction costs) at time $t=1$, with the proceeds of this short 
sale $(0.502917 \times 9.9)$ acquire the American call for 2.435125 , and keep the remaining 2.54375 in the cash account. If the stock price moves up at time $t=1$, exercise the option to collect 5 , and using the cash position coming from node 0 , close the short position. If the stock moves down, do not exercise, close the short position from node 0 , and acquire a new short position in the stock of the order of $1 / 3$ shares to be closed at time $t=2$. This leaves $1 \frac{1}{3}$ in cash. If the stock moves up to 14 , exercise the option, and with the total cash close the short position in the stock. If the stock price moves down to 4 , just close the short position using the available cash.

In the case where the stock makes dividend payments $D_{n}$ at node $n$, model $P^{1}(\lambda, \mu)$ is easily modified, and an extension of Theorem 1 can be proved. This extension is left as an exercise.

In the next section we investigate a relaxation of $P^{1}(\lambda, \mu)$ in connection with randomized stopping times.

\section{Randomized Stopping Times and Relaxation}

Chalasani and Jha (2001) (Sect. 9) and Pennanen and King (2006) obtained pricing expressions for the seller of an ACC in terms of randomized stopping times. A randomized stopping time (Baxter and Chacon 1977; Chalasani and Jha 2001) is a non-negative adapted process (in our case, node function) $Z$ with the property that on every path $\omega$ one has

$$
\sum_{t=0}^{T} Z\left(\omega_{t}\right)=1
$$

That is, the sum of random variables $Z_{0}, Z_{1}, \ldots, Z_{T}$ is equal to 1 on every path. When a randomized stopping time $Z$ is used to describe an exercise strategy, we can think of the value $Z_{n}$ at node $n$ as the probability of exercise at node $n$ given that node $n$ has been reached.

Stopping times are degenerate randomized stopping times. A stopping time $\tau$ corresponds to the randomized stopping time $Z^{\tau}$ whose values are restricted to lie in the set $\{0,1\}$ and defined as follows for any $\omega \in \Omega$, and $t \in\{0,1, \ldots, T\}$ :

$$
Z^{\tau}\left(\omega_{t}\right)= \begin{cases}1 & \text { if } \tau(\omega)=t \\ 0 & \text { otherwise }\end{cases}
$$

The ordinary (or pure) stopping times are extreme points of the convex set of randomized stopping times, or the set $\mathcal{Z}$ of randomized stopping times is the convex hull of the set $\mathcal{T}$ stopping times.

In our setting the set $\tilde{E}$ of randomized stopping times corresponds to the set of $e_{n}$ such that $e_{n} \in[0,1]$ for all $n \in \mathcal{N}$ satisfying the inequalities (3). The practical meaning of passing from stopping times to randomized stopping times as allowable exercise strategies is the possibility of different exercise times for a portfolio of identical ACCs. For a single ACC, a randomized stopping time based exercise strategy can be interpreted as the probabilities of exercise at nodes $n$ with a fractional $e_{n}$ value. 
Chalasani and Jha also proposed in Remark 12.3 of Chalasani and Jha (2001) a formula for the lower hedging price using randomized stopping times. The use of randomized stopping times in the hedging policy as advocated by Chalasani and Jha (2001) implies the following linear programming relaxation $P^{2}(\lambda, \mu)$ of $P^{1}(\lambda, \mu)$ :

$$
\begin{aligned}
\max \quad-b_{0}+ & \sum_{t=1}^{T}\left(d_{0}^{t} S_{0}(1-\mu)-u_{0}^{t} S_{0}(1+\lambda)\right)+e_{0} F_{0} \\
\text { s.t. } \quad b_{n} \leq & R b_{\pi(n)}+e_{n} F_{n}+\sum_{m \in \mathcal{A}(n)} S_{n}\left(u_{m}^{t(n)}-d_{m}^{t(n)}\right) \\
& +\sum_{t=t(n)+1}^{T} S_{n}\left((1-\mu) d_{n}^{t}-(1+\lambda) u_{n}^{t}\right), \quad \forall n \in \overline{\mathcal{N}}, \\
0 \leq & R b_{\pi(n)}+e_{n} F_{n}+\sum_{m \in \mathcal{A}(n)} S_{n}\left(u_{m}^{T}-d_{m}^{T}\right), \quad \forall n \in \mathcal{N}_{T} \\
1 \geq & \left.\sum_{\substack{m \in \mathcal{A}(n) \cup\{n\} \\
e_{n} \in}} e_{m} \forall n \in \mathcal{N}, 1\right], \forall n \in \mathcal{N}
\end{aligned}
$$

and the non-negativity constraints on all the variables $u_{n}^{t}, d_{n}^{t}$, for all $n \in \mathcal{N}$ and all $t \in[0,1, \ldots, T]$. In other words, the relaxation $P^{2}(\lambda, \mu)$ leads to a new price $h_{\text {low }}^{\prime}(\lambda, \mu, F):=\operatorname{opt}\left(P^{2}(\lambda, \mu)\right)$. Chalasani and Jha in Remark 12.3 of Chalasani and Jha (2001) hinted that a relaxation of $h_{\text {low }}(\lambda, \mu, F)$ based on randomized stopping times yields the same value as $h_{\text {low }}(\lambda, \mu, F)$. They did not give an explicit formulation nor a proof of this statement. However, in our relaxation using randomized stopping times, one cannot in general expect to find an integer optimal hedge policy by solving the relaxed problem, i.e., $h_{\text {low }}(\lambda, \mu, F)$ can be smaller than $h_{\text {low }}^{\prime}(\lambda, \mu, F)$. To see this it suffices to go back to the small example of Sect. 2. When we solve this example as a linear program, we obtain an optimal value equal to 2.450000 , which is higher than the value we obtained earlier. This higher value is obtained by the following fractional exercise policy: $2 / 3$ exercise at node 1 , and $1 / 3$ exercise at node 3 or node 4 , and full exercise at node 5 as before.

We have other examples that we do not report to keep the exposition short. On the other hand, in all computational experience, the linear programming relaxation is either exact, or leads to very small duality gaps that are easily closed by off-the-shelf state-of-the-art solvers.

It is clear from the example above that it may be beneficial to the holder of a portfolio of identical ACCs to exercise portions of the portfolio at different time points.

In the frictionless case, i.e., when $\lambda=\mu=0$, the linear programming relaxation $P^{2}(0,0)$ of $P^{1}(0,0)$ yields the same optimal value as $P^{1}(0,0)$. The proof, while quite involved, is a modification of the proof of Theorem 1 in Camc1 and Pinar (2009), hence omitted.

Theorem 2 The optimal value of $P^{2}(0,0)$ is equal to the optimal value of $P^{1}(0,0)$. Furthermore, there exists an optimal solution to $P^{2}(0,0)$ with $e_{n} \in\{0,1\}, \forall n \in \mathcal{N}$. 
The above result implies the formula

$$
h_{\text {low }}(0,0, F)=\max _{Z \in \mathcal{Z}} \min _{\mathbb{Q} \in \mathcal{Q}(0,0)} \mathbb{E}^{\mathbb{Q}}\left[F_{Z}^{*}\right]
$$

Following the same proof technique as in Theorem 4 of Pennanen and King (2006) we can also interchange the max and the min in the above expression, and replace randomized stopping times with ordinary stopping times as a result of the theorem above. Notice that $\mathcal{Q}(0,0)$ coincides with the set of measures $\mathcal{M}$ that make the stock price process a martingale (Chalasani and Jha 2001; King 2002; Pennanen and King 2006). Hence, in an arbitrage free market we obtain:

$$
h_{\text {low }}(0,0, F)=\min _{\mathbb{Q} \in \mathcal{M}} \max _{Z \in \mathcal{Z}} \mathbb{E}^{\mathbb{Q}}\left[F_{Z}^{*}\right]
$$

Finally, the theorem remains valid in the presence of dividend payments as can be routinely verified.

Acknowledgements This research is partially supported by Turkish Scientific and Technological Research Council TUBITAK Grant 107K250, and a senior research scholarship from the United States Fulbright Commission.

\section{References}

Baxter, S., \& Chacon, R. (1977). Compactness of stopping times. Zeitschrift für Die Wahrscheinlichkeit Und Verwandte Gebiete, 40, 169-181.

Bensoussan, A. (1984). On the theory of option pricing. Acta Applicanda Mathematica, 2, 139-158.

Bouchard, B., \& Temam, E. (2005). On the hedging of American options in discrete time markets with proportional transaction costs. Electronic Journal of Probability, 10(22), 746-760.

Buckdahn, R., \& Hu, Y. (1998). Pricing of American contingent claims with jump stock price and constrained portfolios. Mathematics of Operations Research, 23, 177-203.

Camcı, A., \& Pınar, M.Ç. (2009). Pricing American contingent claims by stochastic linear programming. Optimization, 58, 627-640.

Chalasani, P., \& Jha, S. (2001). Randomized stopping times and American option pricing with transaction costs. Mathematical Finance, 11, 33-77.

Davis, M., \& Zariphopoulou, T. (1995). American options and transaction fees. In M. Davis (Ed.), The IMA Volumes in mathematics and its applications (Vol. 65, pp. 47-62). Berlin: Springer-Verlag.

Detemple, J.(2005). American style derivatives: Valuation and computation. Boca Raton: Chapman \& Hall/CRC Financial Mathematics Series.

Edirisinghe, C., Naik, V., \& Uppal, R. (1993). Optimal replication of options with transaction costs and trading restrictions. Journal of Financial and Quantitative Analysis, 28, 117-138.

Föllmer, H., \& Schied, A. (2004). Stochastic finance: An introduction in discrete time. De Gruyter studies in mathematics (2nd ed., Vol. 27). Berlin: W. De Gruyter and Co.

Harrison, J., \& Kreps, D. (1979). Martingales and arbitrage in multiperiod security markets. Journal of Economic Theory, 20, 381-408.

Jaschke, S. (1996). Super-hedging and arbitrage pricing in markets with transaction and trading constraints. Discussion paper 1998-11, SFB 373, Humboldt-Universität Berlin.

Karatzas, I. (1988). On the pricing of American options. Applied Mathematics and Optimization, 17, 37-60.

Karatzas, I., \& Kou, S.G. (1998). Hedging American contingent claims with constrained portfolios. Finance and Stochastics, 2, 215-258.

King, A.J. (2002). Duality and martingales: A stochastic programming perspective on contingent claims. Mathematical Programming Series B, 91, 543-562. 
Koehl, P.-F., Pham, H., \& Touzi, N. (1999). Hedging in discrete-time under transaction costs and continuus-time limit. Journal of Applied Probability, 36, 163-178.

Myeni, R. (1992). The pricing of the American option. Annals of Applied Probability, 2, 1992.

Ortu, F. (2001). Arbitrage, linear programming and martingales in securities markets with bid-ask spreads. Decisions in Economics and Finance, 24, 79-105.

Pennanen, T., \& King, A. (2006). Arbitrage pricing of American contingent claims in incomplete markets - a convex optimization approach. Working paper, June 2006. http://math.tkk.fi/ teemu/ american.pdf.

Roux, A., \& Zastawniak, T. (2006). American options under proportional transaction costs: Seller's price algorithm, hedging strategy and optimal stopping. Preprint, University of York.

Tokarz, K., \& Zastawniak, T. (2006). American contingent claims under small proportional transaction costs. Journal of Mathematical Economics, 43, 65-85. 